\title{
Title: Ultrafast Long-Range Charge Separation in Organic Semiconductor Photovoltaic Diodes
}

\author{
Authors: Simon Gélinas ${ }^{1}$, Akshay Rao ${ }^{1}$, Abhishek Kumar ${ }^{1}$, Samuel L. Smith ${ }^{1}$, Alex W. Chin ${ }^{1}$, Jenny \\ Clark $^{1}$, Tom S. van der Poll ${ }^{2}$, Guillermo C. Bazan ${ }^{2}$, Richard H. Friend ${ }^{1 *}$
}

\author{
Affiliations: \\ ${ }^{1}$ Cavendish laboratory, University of Cambridge, Cambridge, UK \\ ${ }^{2}$ Center for Polymers and Organic Solids, University of California, Santa Barbara, USA \\ *Correspondence to: rhf10@ cam.ac.uk
}

\begin{abstract}
Understanding the charge separation mechanism in organic photovoltaic cells (OPVs) could facilitate optimization of their overall efficiency. Here we report the time dependence of the separation of photogenerated electron hole pairs across the donor-acceptor heterojunction in OPV model systems. By tracking the modulation of the optical absorption due to the electric field generated between the charges, we measure $\sim 200 \mathrm{meV}$ of electrostatic energy arising from electronhole separation within $40 \mathrm{fs}$ of excitation, corresponding to a charge separation distance of at least 4 nm. At this separation, the residual Coulomb attraction between charges is at or below thermal energies, so that electron and hole separate freely. This early time behavior is consistent with charge separation through access to delocalized $\pi$ electron states in ordered regions of the fullerene acceptor material.
\end{abstract}


Main Text: Organic photovoltaic cells (OPVs) consist of a nanostructured blend of donor (D) and acceptor (A) semiconductors $(1,2)$. Photons absorbed in either material create molecular excitons, which can dissociate at the D-A heterojunction into holes on $\mathrm{D}$ and electrons on $\mathrm{A}(3,4)$. The hole and electron are still subject to their mutual Coulomb interaction and can self trap at the heterojunction(58), giving rise to charge transfer (CT) excitons. However, in efficient OPV blends that use fullerenes as the acceptor, electron and hole escape from the heterojunction and long-range charge separation is efficient $(9,10)$ (figure 1A). The motion of charges away from the heterojunction had been generally attributed to diffusion $(11,12)$; however recent results have suggested that delocalized states may play a role in this process $(9)$. Here we directly measure the electron-hole separation process at the heterojunction and find that the Coulomb barrier is surmounted at times as short as $40 \mathrm{fs}$, suggesting that rapid charge motion away from the interface through delocalized band states is a necessary ingredient for long-range charge separation.

In order to temporally resolve the electron-hole separation process we require a probe that is sensitive to the distance between these charges. The electric field generated as the charges separate (figure 1A)(13-15) serves this purpose by shifting the energy levels of neighboring molecules, causing a change in their electronic transition energies and an associated optical electro-absorption (EA)(16) signature, represented schematically in figure $1 \mathrm{~B}(17)$. We measured these EA signals with sub $30 \mathrm{fs}$ precision using transient absorption (TA) spectroscopy. This allows us to calculate the energy stored in the electric field as the charges separate and hence the mean electron-hole distance as a function of time, as we can calibrate the time-resolved data against steady state EA measurements.

We studied two high efficiency model systems. The first consists of blends of a solution-processable small molecule(18), p-DTS(FBTTh 2$)_{2}, \quad$ ((7,7'-(4,4-bis(2-ethylhexyl)-4H-silolo[3,2-b:4,5b']dithiophene-2,6-diyl)bis(6-fluoro-4-(5'-hexyl-[2,2'-bithiophen]-5-yl)benzo[c][1,2,5] thiadiazole))), as electron donor with $\mathrm{PC}_{71} \mathrm{BM}$, (3'H-Cyclopropa[8,25][5,6]fullerene-C $70-\mathrm{D} 5 \mathrm{~h}(6)-3^{\prime}-$ butanoic acid, 3'-phenyl-, methyl ester), as acceptor(19). The second system consists of blends of the polymer PCDTBT(20), (poly[N-11"-henicosanyl-2,7-carbazole-alt-5,5-(40,70- di-2-thienyl-20 ,10 ,30-benzothiadiazole)], as electron donor with $\mathrm{PC}_{61} \mathrm{BM}$, (3'H-Cyclopropa[8,25][5,6]fullerene-C $\mathrm{C}_{60^{-}}$ D5h(6)-3'-butanoic acid, 3'-phenyl-, methyl ester), as acceptor. Figure 1C, D and 1E show their molecular structure and absorption spectra.

The small molecule fullerene blend system (p-DTS(FBTTh $\left.)_{2}: \mathrm{PC}_{71} \mathrm{BM}\right)$ was chosen as it exhibits sharper optical transitions than literature-standard polymer-fullerene blends. As we develop below, this property leads to a strong EA response that enables us to separate this feature from the other 
excited state absorption features. We investigated two blends with different donor/acceptor composition, 60:40 and 90:10. Blends containing a 60:40 weight ratio of p-DTS(FBTTh $)_{2}: \mathrm{PC}_{71} \mathrm{BM}$, processed from chlorobenzene with $0.4 \%$ diiodooctane (DIO) as a solvent additive, achieve very high internal quantum efficiency (IQE), and power-conversion efficiencies (PCE) above 7\% $(19,21)$. They have been shown to comprise crystalline regions of $\mathrm{p}$-DTS(FBTTh$)_{2}$ and $\mathrm{PC}_{71} \mathrm{BM}$ aggregates(21). We contrast this system with the 90:10 blend, processed from chloroform, which shows much lower quantum efficiency and for which the low fullerene fraction precludes aggregation of the fullerene $($ See SM)(22-24).

PCDTBT is a widely studied non-crystalline polymer, which when blended with PCBM gives IQEs close to $100 \%$ and PCEs as high as $7 \%(25,26)$. As has been discussed previously, PCBM effectively intercalates into the polymer side chains. Thus at low fullerene loading the PCBM is mainly unaggregated, forming a mixed phase with the polymer(23). As the fullerene content is increased the excess fullerene begins to form aggregates(27). Here we contrast a low PCBM loading, 20\% (4:1), which does not show fullerene aggregation, to the optimized $80 \%(1: 4)$ loading that shows high quantum efficiencies and gives rise to aggregated fullerene domains.

Figures 2A-C show TA data for the 90:10 and 60:40 p-DTS(FBTTh $)_{2}: \mathrm{PC}_{71} \mathrm{BM}$ blends covering the visible and near-infrared spectrum from 100 fs to $2 \mathrm{~ns}$. The positive features between 600 and 700nm are due to bleaching of the ground state absorption features (GSB). The negative signal peaking at $1230 \mathrm{~nm}$ (extending up to $1500 \mathrm{~nm}$ ) is due to the photoinduced absorption (PIA) of singlet excitons, in agreement with measurements on pristine films of p-DTS(FBTTh $)_{2}$ (see Fig. S2). The negative signal that peaks at $800 \mathrm{~nm}$ is due to the PIA from positive charges (polarons) on the donor. Its amplitude is similar for both blends, indicating that the density of photogenerated holes is comparable. However, the subsequent behavior is very different. In comparison to the 90:10 blend, the 60:40 blend shows a more complex behaviour around $710 \mathrm{~nm}$. The strength of the bleach is reduced at early times, and a negative response is observed beyond $10 \mathrm{ps}$. This is clearly seen (Fig. 2D) in the spectra at 100 ps delay of the two blends, of a neat film of p-DTS(FBTTh $)_{2}(100: 0)$, and of the steady-state electro-absorption spectra of p-DTS(FBTTh $)_{2}$ (measured independently, see Fig. S7). When compared to the neat film, the 90:10 blend shows a reduction of stimulated emission (SE) at wavelengths $>720 \mathrm{~nm}$, indicating efficient exciton quenching. In addition, the 60:40 blend also shows a large negative signal peaking at $710 \mathrm{~nm}$, which matches the measured steady-state EA spectrum in this region. 
From the data presented in figure 2, we identify two time-scales for charge generation. We consider that the early sub-ps dynamics are due to the dissociation of excitons photogenerated at the heterojunction, whereas the later evolution is due to excitons photogenerated in bulk pDTS(FBTTh $)_{2}$ domains which diffuse before reaching heterojunctions where they dissociate. We use a global analysis to separate spectral features that have different time evolutions (see SM). Figure 3 A shows the separation of the different features in the 60:40 blend over the time range 30 to 2500 fs. The evolution of the singlet exciton and hole absorption spectra is described by two time constants of $82 \pm 5$ fs and $22 \pm 0.1$ ps (with $70 \%$ weight to the 82 fs component); the exciton component decreases and the charge component grows with time as shown in figure 3B. Our global analysis reveals a third feature with distinct temporal dynamics. This feature closely resembles the steady-state EA spectrum, as shown in figure $3 \mathrm{~A}$, confirming the presence of an EA signature in the 60:40 blend. The singlet spectrum in figure $3 \mathrm{~A}$ is in good agreement with that of neat p-DTS(FBTTh $)_{2}$ films in figure $2 \mathrm{D}$, where the signal is due only to singlet excitons. The signature of the hole polaron matches the spectrum of the 90:10 blend (figure 2D) but differs substantially from the 60:40 blend, where features due to both holes and EA are present. This comparison indicates that charge generation occurs in the 90:10 blend without inducing an EA signature, in contrast to the 60:40 blend.

Turning to the dynamics of the EA feature, figure 3B, we note that some oscillations are present within the first picosecond, which are due to a non-resonant artefact, and this limits our reconstruction of the EA kinetics to delays $\geq 300$ fs. At $300 \mathrm{fs}$, we already observe a large signal, more than half of the value at $2 \mathrm{ps}$, beyond which the signal saturates. At low temperatures $(4 \mathrm{~K})$ the early time response is the same, but the later growth is delayed, see Fig. S4. As noted above, the EA feature is absent from the 90:10 blends where charges are generated efficiently but are unable to separate away from the heterojunction. This implies that the EA of a barely-separated charge-pair is negligible, and hence the large EA-signal occurs due to longer range charge separation. The EA signature in the 60:40 blend thus allows us to resolve the separation of electron-hole pairs.

Figure 3D shows the spectral signatures of singlet and charge features in 4:1 and 1:4 blends of PCDTBT:PCBM. The spectral signatures of charges in the two blends differ significantly, with the 1:4 blend having a more pronounced negative feature at $640 \mathrm{~nm}$. This is qualitatively similar to the behavior of the p-DTS(FBTTh $)_{2}: \mathrm{PC}_{71} \mathrm{BM}$ blends where the 60:40 blend, which shows EA, has a more pronounced negative feature at 710nm than the 90:10 blend which does not show EA. Using the difference between the 4:1 and 1:4 PCDTBT:PCBM blends allows us to extract a third spectral component for the 1:4 blend (purple in Fig. 3D; see SM for details)that matches the steady-state EA spectrum. The dynamics of the blends are shown figure $3 \mathrm{C}$ : the singlet exciton is rapidly quenched 
(86 fs) by charge transfer in both the $4: 1$ and 1:4 blends, with corresponding growth in the charge population. There is no EA response for the 4:1 blend, as observed in figure 3D, but the 1:4 blend shows a large EA signal at $40 \mathrm{fs}$. For this system the non-resonant artefact is not as pronounced as for the p-DTS(FBTTh $)_{2}: \mathrm{PC}_{71} \mathrm{BM}$ allowing accurate EA detection at delays as short as $40 \mathrm{fs}$.

By performing quasi-steady-state electro-absorption measurements, which measure the EA signature induced by an oscillating electric field on p-DTS(FBTTh $)_{2}: \mathrm{PC}_{71} \mathrm{BM}$ and PCDTBT:PCBM diode devices, we can calibrate the EA amplitude to the electric field present in the semiconductor layer (See SM for details). Using this calibration, we convert the transient EA amplitude of Figure 3 to a spatially-averaged value of the square of the electric field. We calculate that the RMS field strength and hence that the energy stored in the electric field, $\int \frac{\varepsilon \varepsilon_{0}}{2} E^{2} d V$.

Figure 3E shows the energy stored in the electric field per charge pair as a function of time for the various blends studied (taking a value of 3.5 for $\varepsilon$ and modelling half the field to be present in the donor phase). For the $60: 40$ p-DTS(FBTTh $)_{2}: \mathrm{PC}_{71} \mathrm{BM}$ the energy per charge pair reaches more than half its maximum value by 300fs and for the 1:4 PCDTBT:PCBM blends it attains its saturation value by $40 \mathrm{fs}$. The energies involved are substantial, well above $\mathrm{k}_{\mathrm{B}} \mathrm{T}$, and provide direct evidence that considerable work must be done to overcome the Coulomb attraction between the separating electron hole pairs in these low dielectric constant materials. Crucially, the majority of this work against the Coulomb interaction is done on ultrafast timescales. This requires that charges underdo rapid spatial separation on these timescales.

With simple electrostatic modelling and the assumption that the initial step of photoinduced electron transfer just across the heterojunction causes an average separation of electron and hole by $1.5 \mathrm{~nm}$ in the absence of fullerene aggregation, we find that charges reach a separation of 4 to $5 \mathrm{~nm}$ in both systems at the earliest times measured (see SM). The further growth for the 60:40 p$\mathrm{DTS}\left(\mathrm{FBTTh}_{2}\right)_{2}: \mathrm{PC}_{71} \mathrm{BM}$ blend on time scales greater than $300 \mathrm{fs}$ is most likely due to the diffusive motion of the hole within the crystalline p-DTS(FBTTh $\left.)_{2}\right)_{2}$ domains. For PCDTBT this is not observed as the larger fullerene domains lead to an increased initial separation, resulting in the saturation of the EA signal (This occurs when the separation reaches 5 to $6 \mathrm{~nm}$ : see SM for details).

The length scales being considered here for the separation of charges, $\approx 4$ to $5 \mathrm{~nm}$, are consistent with known OPV morphology in efficient systems, where the presence of pure domains of fullerene, $5 \mathrm{~nm}$ in length $(28,29)$, is strongly correlated with suppressed geminate recombination and improved device performance. In our measurements the presence of fullerene aggregates leads to large EA signals and 
electrostatic energies at short times, strongly suggesting that they are key to enabling ultrafast longrange charge separation.

Our measurements are particularly sensitive to the early-time separation of charge carriers at heterojunctions, due to our direct measurement of $\left|\mathbf{E}^{2}\right|$ which is insensitive to the initial direction of charge separation with respect to charge collection electrodes (which we expect to be randomly directed, given the bulk heterojunction morphology of these materials). This initial process in not dependent on the presence of an external electric field. In contrast, other time-resolved techniques such as EFISH(30) are sensitive to a change in $|\mathbf{E}|$. In such experiments this change in $|\mathbf{E}|$ arises when carriers drift in the field set up by the electrodes and the external circuit. This necessarily builds up at later times. For example, Vithanage et al.(30) use EFISH to measure net charge motion due to drift towards the electrodes of $2 \mathrm{~nm}$ at $1 \mathrm{ps}$ and $5 \mathrm{~nm}$ at $100 \mathrm{ps}$. This provides complementary information to our measurements of the first steps in charge separation. See SM for details.

Based on the above results we propose a simple phenomenological model of the heterojunction interface. We recall that films without aggregated fullerene domains do not exhibit a substantial EA response; which implies that the mobile component of the charge pair is the electron at early times. We model the electron motion on a nanoscale FCC lattice of acceptor sites, consisting of localized single electron energy levels which are coherently coupled to their nearest neighbors (a tight binding model) giving rise to a band of delocalized eigenstates, with bandwidth B. We include a Gaussian distribution of acceptor site energies to introduce disorder. As expected, we find that when the static disorder of the on-site energies is within the bandwidth, disorder does not localize the states. The initial excitation is a single exciton on an adjacent donor site.

We include a Coulomb well, of depth $\mathrm{W}$, surrounding the donor to model the presence of the hole, which we assume does not move during the first 200fs of charge separation. The well lowers the energies of electron states in the local vicinity of the donor (Fig. 4A), introducing a set of bound states and narrowing the energetic width of delocalised states to $\approx \mathrm{B}-\mathrm{W}$. Typical structures of the emergent eigenstates are illustrated in Fig. 4A ( $\left.\varphi_{1-2}\right)$, with corresponding examples of the actual states given in Fig. S11. We emphasize that our model is only valid for a few hundred fs after exciton dissociation when delocalized stares are briefly accessed; after this, polaron formation will localize the electronic states and holes and electrons will move via incoherent hopping with comparable mobilities. 
If the energy of the electron on the donor site lies within the spread of fullerene eigenstates at the interface, the electron can undergo resonant transfer. When this energy lies below the conduction edge (CE, Fig. 4A), the initial electron wave function can only mix with bound states localized near the hole $\left(\varphi_{1}\right)$, forming a bound charge-transfer state. However if the initial electron energy lies within the conduction channel above this edge, then the initial state is mixed with states that are fully delocalized across the fullerene lattice $\left(\varphi_{2}\right)$; enabling long range charge separation to occur.

The model described above assumes spatial coherence between acceptor sites (delocalization). In order to describe the dynamics of the system, we consider two cases: (i) incoherent transitions between a localized donor site and the delocalized acceptor eigenstates, or (ii) a fully coherent case where the initial electron is described as phase-coherent superposition of these delocalised eigenstates. We perform simulations for both these models on a $5.25 \times 5.25 \times 5.25 \mathrm{~nm}$ lattice of acceptor sites and for a range of couplings and disorder strengths between 30 to $50 \mathrm{meV}$ and 0.1 to $0.2 \mathrm{eV}$ respectively $(31,32)$; all parameters are described in full in SM.

Incoherent transitions arise from a perturbative treatment of the donor-acceptor coupling (case i, see $\mathrm{SM}$ ). This generates a set of Fermi golden rule transition rates from the localized donor site into the delocalized states described earlier. Results are shown in figure 4B, yellow curves. We find that charge separation occurs via a single hop of $\sim 4 \mathrm{~nm}$. For case ii, we evolve the coherent superposition under the Schrödinger equation, which generates a wavepacket that rapidly propagates across the Coulomb well and into the acceptor crystallite (see SM for details). Results are shown in figure 4B, dark red curves. Again for all parameter values considered, electron and hole separate by 3 to $5 \mathrm{~nm}$ within 300 fs. For both cases the separation distance is determined primarily by the size of the acceptor aggregate in our model. Currently our experiments cannot differentiate between these two separation mechanisms.

We also consider charge dynamics under fully incoherent localized dynamics (Marcus theory), i.e. in the absence of delocalization, for the same parameters (See SM for details). This leads to rapid exciton dissociation, with hole and electron on nearest neighbour sites as shown in Fig. 4b, blue curve to form tightly bound CT states that are not expected to dissociate rapidly.

Our demonstration and model of short-time charge delocalization and coherent motion is very different from models for exciton coherence and delocalization that have been suggested as being key 
to efficient OPV performance(33). Within our study we find no need to invoke such excitonic processes; see SM for details.

Our results rationalize the apparent asymmetry between efficient electron-hole capture in organic light-emitting diodes(34) and near-unity photo-conversion quantum efficiencies in OPVs(25) by revealing that ultrafast charge separation through delocalized band-like states in fullerene aggregates is key to efficient charge separation. Moreover, the fast timescale for this process indicates that efficient charge separation requires no excess energy beyond that needed to overcome the Coulomb interaction. This is in contrast to Onsager-like models that require excess energy in hot states. Our results suggest that the real energy loss during charge separation lies elsewhere, for instance in later energetic relaxation of charges through polaron formation or the presence of defect-mediated gap states and that such energy losses are not fundamental for efficient charge separation. 


\section{Acknowledgements:}

We thank the EPSRC, and the Winton Programme (Cambridge) for the Physics of Sustainability for funding. S.G. acknowledges funding from the Fonds québécois de recherche sur la nature et les technologies, AR thanks Corpus Christi College for a Research Fellowship, AK thanks NRFSingapore for a scholarship, JC thanks the Royal Society for a Dorothy Hodgkin Fellowship and TVP acknowledges funding from the Center for Energy Efficient Materials, an Energy Frontier Research Center funded by the U.S. Department of Energy, Office of Science, Basic Energy Sciences under Award \#DC0001009. 


\section{Reference and Notes:}

1. J. J. M. Halls et al., Efficient photodiodes from interpenetrating polymer networks, Nature 376, 498-500 (1995).

2. G. Yu, J. Gao, J. C. Hummelen, F. Wudl, A. J. Heeger, Polymer Photovoltaic Cells: Enhanced Efficiencies via a Network of Internal Donor-Acceptor Heterojunctions, Science (80-. ). 270, 17891791 (1995).

3. T. M. Clarke, J. R. Durrant, Charge photogeneration in organic solar cells., Chem. Rev. 110, 6736-67 (2010).

4. J.-L. Brédas, J. E. Norton, J. Cornil, V. Coropceanu, Molecular Understanding of Organic Solar Cells: The Challenges, Acc. Chem. Res. 42, 1691-1699 (2009).

5. X.-Y.-Y. Zhu, Q. Yang, M. Muntwiler, Charge-transfer excitons at organic semiconductor surfaces and interfaces., Acc. Chem. Res. 42, 1779-87 (2009).

6. A. E. Jailaubekov et al., Hot charge-transfer excitons set the time limit for charge separation at donor/acceptor interfaces in organic photovoltaics, Nat. Mater. 11, 1-8 (2012).

7. S. Gélinas et al., The Binding Energy of Charge-Transfer Excitons Localized at Polymeric Semiconductor Heterojunctions, J. Phys. Chem. C 115, 7114-7119 (2011).

8. R. D. Pensack, J. B. Asbury, Beyond the Adiabatic Limit: Charge Photogeneration in Organic Photovoltaic Materials, J. Phys. Chem. Lett. 1, 2255-2263 (2010).

9. A. A. Bakulin et al., The role of driving energy and delocalized States for charge separation in organic semiconductors., Science (80-. ). 335, 1340-4 (2012).

10. G. Grancini et al., Hot exciton dissociation in polymer solar cells, Nat. Mater. 11, 1-5 (2012).

11. P. W. M. Blom, V. D. Mihailetchi, L. J. A. Koster, D. . Markov, Device Physics of Polymer: Fullerene Bulk Heterojunction Solar Cells, Adv. Mater. 19, 1551-1566 (2007).

12. D. Caruso, A. Troisi, Long-range exciton dissociation in organic solar cells., Proc. Natl. Acad. Sci. U. S. A. 109, 13498-502 (2012).

13. J. Cabanillas-Gonzalez et al., Subpicosecond photoinduced Stark spectroscopy in fullerenebased devices, Phys. Rev. B 75, 1-7 (2007).

14. A. Devižis, A. Serbenta, K. Meerholz, D. Hertel, V. Gulbinas, Ultrafast Dynamics of Carrier Mobility in a Conjugated Polymer Probed at Molecular and Microscopic Length Scales, Phys. Rev. Lett. 103, 027404 (2009).

15. J. Cabanillas-Gonzalez et al., Photoinduced Transient Stark Spectroscopy in Organic Semiconductors: A Method for Charge Mobility Determination in the Picosecond Regime, Phys. Rev. Lett. 96, 6-9 (2006).

16. L. Sebastian, G. Weiser, H. Bässler, Charge transfer transitions in solid tetracene and pentacene studied by electroabsorption, Chem. Phys. 61, 125-135 (1981).

17. I. Campbell, T. Hagler, D. Smith, J. Ferraris, Direct Measurement of Conjugated Polymer Electronic Excitation Energies Using Metal/Polymer/Metal Structures, Phys. Rev. Lett. 76, 19001903 (1996).

18. Y. Sun et al., Solution-processed small-molecule solar cells with $6.7 \%$ efficiency., Nat. Mater. 11, 44-8 (2012).

19. T. S. van der Poll, J. A. Love, T.-Q. Nguyen, G. C. Bazan, Non-basic high-performance molecules for solution-processed organic solar cells., Adv. Mater. 24, 3646-9 (2012).

20. N. Blouin, A. Michaud, M. Leclerc, A Low-Bandgap Poly(2,7-Carbazole) Derivative for Use in High-Performance Solar Cells, Adv. Mater. 19, 2295-2300 (2007). 
21. J. A. Love et al., Film Morphology of High Efficiency Solution-Processed Small-Molecule Solar Cells, Adv. Funct. Mater. , n/a-n/a (2013).

22. A. A. Bakulin, J. C. Hummelen, M. S. Pshenichnikov, P. H. M. van Loosdrecht, Ultrafast HoleTransfer Dynamics in Polymer/PCBM Bulk Heterojunctions, Adv. Funct. Mater. 20, 1653-1660 (2010).

23. a. C. Mayer et al., Bimolecular Crystals of Fullerenes in Conjugated Polymers and the Implications of Molecular Mixing for Solar Cells, Adv. Funct. Mater. 19, 1173-1179 (2009).

24. L. G. Kaake, Y. Sun, G. C. Bazan, A. J. Heeger, Fullerene concentration dependent bimolecular recombination in organic photovoltaic films, Appl. Phys. Lett. 102, 133302 (2013).

25. S. H. Park et al., Bulk heterojunction solar cells with internal quantum efficiency approaching 100\%, Nat. Photonics 3, 297-302 (2009).

26. Z. He et al., Simultaneous enhancement of open-circuit voltage, short-circuit current density, and fill factor in polymer solar cells., Adv. Mater. 23, 4636-43 (2011).

27. N. C. Miller et al., Molecular packing and solar cell performance in blends of polymers with a bisadduct fullerene., Nano Lett. 12, 1566-70 (2012).

28. B. a. Collins et al., Absolute Measurement of Domain Composition and Nanoscale Size

Distribution Explains Performance in PTB7:PC 71 BM Solar Cells, Adv. Energy Mater. 3, 65-74 (2013).

29. F. Liu, Y. Gu, J. W. Jung, W. H. Jo, T. P. Russell, On the morphology of polymer-based photovoltaics, J. Polym. Sci. Part B Polym. Phys. 50, 1018-1044 (2012).

30. D. A. Vithanage et al., Visualizing charge separation in bulk heterojunction organic solar cells., Nat. Commun. 4, 2334 (2013).

31. R. C. I. Mackenzie, J. M. Frost, J. Nelson, A numerical study of mobility in thin films of fullerene derivatives., J. Chem. Phys. 132, 064904 (2010).

32. H. Tamura, M. Tsukada, Role of intermolecular charge delocalization on electron transport in fullerene aggregates, Phys. Rev. B 85, 054301 (2012).

33. L. G. Kaake, D. Moses, A. J. Heeger, Coherence and Uncertainty in Nanostructured Organic Photovoltaics, J. Phys. Chem. Lett. 4, 2264-2268 (2013).

34. A. C. Morteani et al., Barrier-free electron-hole capture in polymer blend heterojunction lightemitting diodes, Adv. Mater. 15, 1708 (2003).

35. A. Rao, M. W. B. Wilson, S. Albert-Seifried, R. Di Pietro, R. H. Friend, Photophysics of pentacene thin films: The role of exciton fission and heating effects, Phys. Rev. B 84, 195411 (2011).

36. S. Albert-Seifried, R. H. Friend, Measurement of thermal modulation of optical absorption in pump-probe spectroscopy of semiconducting polymers, Appl. Phys. Lett. 98, 223304 (2011).

37. R. A. Marsh, J. M. Hodgkiss, S. Albert-Seifried, R. H. Friend, Effect of annealing on P3HT:PCBM charge transfer and nanoscale morphology probed by ultrafast spectroscopy., Nano Lett. 10, 923-30 (2010).

38. L. G. Kaake et al., Photoinduced charge generation in a molecular bulk heterojunction material., J. Am. Chem. Soc. 134, 19828-38 (2012).

39. G. Li, B. Movaghar, M. a. Ratner, Dynamic electron localization initiated by particle-bath coupling, Phys. Rev. B 87, 094302 (2013).

40. A. Troisi, How quasi-free holes and electrons are generated in organic photovoltaic interfaces, Faraday Discuss. (2013), doi:10.1039/c3fd20142b. 
41. W. I. F. David et al., Crystal structure and bonding of ordered C60, Nature 353, 147-149 (1991).

42. H. Bruus, K. Flensberg, Many-Body Quantum Theory in Condensed Matter Physics: An Introduction (Oxford University Press, 2004), p. 466.

43. S. Mukamel, Principles of Nonlinear Optical Spectroscopy (Oxford University Press, 1999), p. 576.

44. G. D. Scholes, Insights into excitons confined to nanoscale systems: electron-hole interaction, binding energy, and photodissociation., ACS Nano 2, 523-37 (2008). 
Fig. 1. Schematics of interfacial photophysical processes in OPVs (and molecules studied). (A) Overview of charge photogeneration at a heterojunction. Light absorption generates excitons in the bulk (1a) and at interfaces (1b). When next to an interface, excitons undergo rapid charge transfer into an electron-hole pair (2), generating a dipole-like electric field in its surroundings $(\vec{E})$. The electron and hole then separate further and form free charges (3). (B) Stark shift of the absorption spectrum (S) due to an electric field $\overrightarrow{\mathrm{E}}$ (dark blue) and the resulting electro-absorption signature (red) calculated from the difference between the shifted and un-shifted spectra. The EA amplitude is proportional to $|\vec{E}|^{2}$. (C) Chemical structure of $\mathrm{PC}_{61} \mathrm{BM}$ (grey) and PCDTBT (red). (D) Chemical structure of $\mathrm{PC}_{71} \mathrm{BM}$ (grey) and p-DTS(FBTTh $)_{2}$ (blue). (E) Absorption spectra of the molecules presented in D. 
Fig. 2. Transient absorption spectra of excited states in p-DTS(FBTTh $)_{2}$ :PC P1 $_{71}$ B films. (A, B) Visible and near-infrared measurements of a 60:40 film prepared identically to the active layer of an efficient OPV device. The samples were excited with $700 \mathrm{~nm}$ laser pulses at a fluence of $2 \mu \mathrm{J} / \mathrm{cm}^{2}$. (C) Measurement of a 90:10 film, where $\mathrm{PC}_{71} \mathrm{BM}$ aggregation is prevented and charge separation inefficient. (D) TA time slices of the 100:0, 90:10 and 60:40 films normalized at $640 \mathrm{~nm}$. The steadystate EA signature, obtained from a device using a steady-state electro-absorption measurement, is proportional to the $1^{\text {st }}$ derivative of the absorption spectrum. 


\section{Fig. 3. Excited-state and EA dynamics of p-DTS(FBTTh $\left.)_{2}\right)_{2}: \mathrm{PC}_{71} B M$ and PCDTBT:PC ${ }_{61} B M$}

blends. (A) Spectral signatures present in the p-DTS(FBTTh $)_{2}: \mathrm{PC}_{71} \mathrm{BM}(60: 40)$ blend. The signals are obtained from a global fit spanning the entire visible range (see text for details). The dataset spans 30 to 2500 fs and the sample was excited with broadband (525 to $625 \mathrm{~nm}$ ) laser pulses compressed to $30 \mathrm{fs}$ at a fluence of $8 \mu \mathrm{J} / \mathrm{cm}^{2}$. The reference EA is obtained independently (see figure 2D). (B) Dynamics of the $S_{1}$, hole and EA amplitudes obtained by fitting the dataset with a linear combination of the excited-state signatures shown in A. The yellow zone indicates the region over which artefacts prevent accurate measurement of the EA. (C,D) Spectral signatures and their associated dynamics for three different compositions of PCDTBT:PC ${ }_{61} \mathrm{BM}$ blends, see SM for details of the analysis. The samples were excited at a fluence of $2 \mu \mathrm{J} / \mathrm{cm}^{2}$ and the reference EA spectrum was measured on a device. (E) Total energy stored in the electric field per electron-hole pair (at room temperature and $4 \mathrm{~K})$. The EA is converted to a spatially-integrated electric field square using a calibration from steadystate measurements $\left(E A=C_{E A} \int|E|^{2} d V\right.$, where $C_{\mathrm{EA}}$ is the calibration constant for a given system. See SM for details). The time-resolved energy per hole $\left(\frac{1}{2} \varepsilon \varepsilon_{0} \int|E|^{2} d V\right)$ is obtained assuming that half of the field is in the donor phase. The low temperature $(4 \mathrm{~K})$ measurement grows continuously up to $1 \mathrm{~ns}$ where it saturates at $\sim 200 \mathrm{meV}$ (see SM). 
Fig. 4. Model of initially-accessible electronic states in fullerene derivatives and calculated electron-hole separation distance. (A) Excited states before and immediately after charge transfer. When excitons $\left(\mathrm{S}_{1}\right)$ dissociate at interfaces with aggregated $\mathrm{PC}_{61 / 71} \mathrm{BM}$, the isoenergetic charge transfer places the electrons in delocalized band states, where they undergo wave-like propagation within the $\mathrm{PC}_{61 / 71} \mathrm{BM}$ aggregate. In this model, the hole at the interface induces a well of depth $\mathrm{W}$, reducing the width of the band from its bulk value $\mathrm{B}$ to $\sim \mathrm{B}-\mathrm{W}$. This system can sustain two typical electron wavefunctions represented as $\varphi_{1,2}$. The electron is either trapped at the interface $\left(\varphi_{1}\right)$ or propagating through the band $\left(\varphi_{2}\right)$. (B) Calculation of electron-hole separation dynamics per charge pair for (i) injection of a fully coherent electron wavepacket, (ii) tunnelling of the electron into delocalized states (Fermi Golden rule, FGR), and (iii) tunnelling of the electron into localized states (i.e. Marcus-type electron transfer). The multiple lines represent different values of disorder and couplings, spanning 100 to $200 \mathrm{meV}$ and 30 to $50 \mathrm{meV}$ respectively $(31,32) . r_{0}$ is the separation distance of the initial next-neighbour charge-transfer state (typically $1.5 \mathrm{~nm}(9)$ ) and $l$ is the length of the $\mathrm{PC}_{61 / 71} \mathrm{BM}$ aggregate over which the wavefunction is delocalized $(5.25 \mathrm{~nm}$ for the calculation shown). 